\title{
Combining autologous particulate dentin, L-PRF and fibrinogen to create a matrix for predictable ridge preservation: A pilot clinical study
}

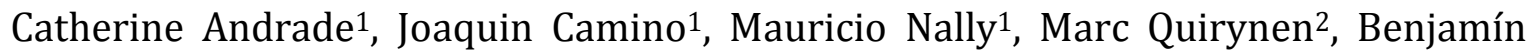
Martínez $^{3}$ \& Nelson Pinto ${ }^{1,2}$

${ }^{1}$ Department of Periodontology and Oral Implantology, Faculty of Dentistry, Universidad de Los Andes, Santiago, Chile.

2Section of Periodontology, Department of Oral Health Sciences, KU Leuven \& Dentistry, University Hospitals, KU Leuven, Kapucijnenvoer 7, 3000 Leuven, Belgium. ${ }^{3}$ Department of Oral Diagnosis and Oral Pathology, School of Dentistry, Universidad Mayor, Santiago, Chile.
\end{abstract}

\section{Corresponding author}

Catherine Andrade, DDS, Department of Periodontology and Oral Implantology, School of Dentistry, Universidad de Los Andes, Santiago, Chile; Monseñor Álvaro del Portillo12455, Las Condes - Santiago, Chile; telephone: +56972862308; E-mail: candrade@uandes.cl.

\section{Acknowledgments}

The authors acknowledge the help and support received from the staff of the Periodontology and Implantology Department of Universidad de Los Andes. 


\section{Abstract}

\section{Objectives}

The aim of this study was to describe the histological and clinical outcome of "Dentin block" (a mixture of: autologous particulate dentin, leukocyte and platelet rich fibrin (L-PRF) and liquid fibrinogen) in an alveolar ridge preservation.

\section{Material and Methods}

10 extraction sockets were grafted with "Dentin Block" a mixture of particulate autologous dentin with chopped leukocyte-platelet rich fibrin (L-PRF) membranes at a 1:1 ratio and liquid fibrinogen as binder. 2 grafted sites were followed at 4 and 5 months, and 6 sites at 6 months. Biopsies were taken from the core of the grafted site for histologic and histomorphometric analysis.

\section{Results}

All patients completed the study without any adverse event. The vertical and horizontal dimensions of alveolar ridge were preserved or even increased after 4, 5 or 6 months and remained stable after 6 months of the implant placement. The histological examination revealed a median relative percentage of bone, dentin, and connective tissue of 57.0, 0.9, and $39.3 \%$, respectively. A comparison of samples at different time points (4, 5, and 6 months) showed a progressive increase in the proportion of bone with a decrease in the proportion of dentin. The bone was compact with normal osteocytes and moderate osteoblastic activity. In $4 / 10$ samples no dentin was observed, in the other samples it represented 1-5\% (with geometric fragments).

\section{Conclusions}

Dentin block showed to be a suitable bone substitute in an alveolar ridges preservation model.

Clinical Relevance: The promising results of Dentin Block as a bone substitute in alveolar ridge preservation could have an important clinical impact considering this biomaterial brings together the regenerative potential of three autologous products with excellent biological and clinical behavior, low risk of adverse effects and feasible acquisition.

\section{Key words}

Bone substitute; bone regeneration; dentin; dentin block; GBR; ridge preservation. 


\section{Introduction}

The alveolar process is prone to major resorption in a vertical and horizontal dimension after tooth extraction $[1,2]$, primarily due to the loss of bundle bone. This may jeopardize future implant therapy [3], which requires an adequate three-dimensional osseous volume of the alveolar ridge, accompanied by a good soft tissue architecture to provide an optimal aesthetic, phonetic and long-term functional result [4].

Various surgical techniques have been proposed to compensate for this resorption. Alveolar ridge preservation (ARP) techniques are widely used to compensate this resorption. The application of grafting biomaterials into a fresh extraction socket has been thoroughly investigated in both animal and clinical studies [5-7]. These materials are used because they can possess one or more of these biological properties: osteogenesis, osteoinduction and/or osteoconduction [8]. A systematic review from Vignoletti and co-workers [9] revealed that there are no clear guidelines on which biomaterial to select for this purpose. A recent metaanalysis highlighted that the resorption of the alveolar ridge cannot be "completely" avoided by ARP [10]. Therefore, further development in the regenerative field remains fundamental to provide new alternatives of biomaterials.

So far autogenous bone is the only graft material with osteogenic properties, and therefore, it is considered the gold standard for bone grafting $[11,12]$. Unfortunately, autogenous bone has limitations related to: availability, morbidity, and risks during the graft harvest $[13,14]$. Bone substitutes (allografts, xenografts, alloplastic materials, etc.) have variable rates of resorption and bone formation, and are associated with a high financial cost [14]. Moreover, graft remnants may decrease the final bone-to-implant contact (BIC) [15].

Since the 60's, dentin has been evaluated as a biomaterial to induce bone formation [16]. To date, several in vitro, in vivo, and clinical studies have reported promising results, proposing dentin as an adequate bone substitute [8, 17-20]. The fact that dentin shares the same embryologic origin as alveolar bone might explain its capacity for bone formation. Moreover, dentin and bone composition are very similar [21]. Dentin organic composition is mainly collagen I (90\%) and it contains various growth factors such as bone morphogenic proteins (BMP's), recognized as promoter of bone formation [21, 22]. Dentin, as a bone substitute, has also the advantage that it does not cause host tissue reaction or heterotopic bone formation, which is an important safety aspect to consider when a graft is selected [23, 24].

In ARP, dentin has shown be able to preserved the dimensions of alveolar ridge due to osteoconductive property and its favorable rate of resorption and new bone formation [25]. Dentin showed already to be an effective bone substitute for vertical augmentation in extraction sockets with results comparable to inorganic bovine bone, with for example similar implant stability [26]. 
To enhance wound healing, the use of biological additives, which regulate inflammation and angiogenesis, could be very beneficial. Choukroun and co-workers (2001) introduced a second generation platelet concentrate (the leucocyte and platelet-rich fibrin (L-PRF)), which is simple in preparation and does not require a biochemical blood handling [27]. The fibrin network has many similarities with the one formed during natural healing. Moreover, the release of high quantities of transforming: growth factor Beta-1 (TGF beta-1), plateletderived growth factor $\mathrm{AB}$ (PDGF-AB), vascular endothelial growth factor (VEGF) and thrombospondin-1 from L-PRF [28] stimulates biological functions, such as chemotaxis, angiogenesis, proliferation, differentiation [29-31].

Several medical disciplines have used L-PRF in different types of surgery, including periodontology, implant dentistry and maxillofacial surgery, reporting a positive effect for the wound healing process [32-41].

Recently, a split-mouth, randomized clinical trial reported significant benefits in the preservation of vertical and horizontal ridge dimensions with the use of leukocyte-platelet rich fibrin (L-PRF) as "sole" grafting material. It showed similar results in ARP as other bone substitutes, with several advantages such as: simplified procedures, less post-operatory pain and inflammation, the absence of residual graft particles and the benefit of its low cost [44]. These benefits of L-PRF in the healing time are confirmed by a systematic review showing that ARP improves when bone substitutes are combined with L-PRF [8].

On the other hand, a new concept, "liquid fibrinogen", has been introduced as a binder to particulate bone graft, allowing improve its stabilization in the defect and adding a potential biological effect by their cellular content, which could accelerate the healing process and optimize the new bone formation [45].

The aim of the present pilot study was to describe, for the first time as far as the authors know, the clinical and histological outcome of a "Dentin block" (a mixture of: $50 \%$ autogenous dentin, $50 \%$ L-PRF membranes chopped into small pieces and liquid fibrinogen to agglutinate all together for better handling) as a bone substitute using alveolar ridge preservation as test model.

\section{Material and methods}

\section{Patient selection}

Four patients with a total of 10 extraction sockets at locations scheduled for future implant rehabilitation, were enrolled in this pilot study. The patients met the following inclusion criteria: older than 18 years, no medical history that contraindicates the surgical procedure, at least one tooth that had to be extracted but without endodontic treatment, post-extraction socket with 4 bone walls, request for replacement by an implant. The exclusion criteria were: systemic disease that might impair bone metabolism, antiresorptive therapy (as bisphosphonates), pregnancy, psychiatric conditions and heavy smokers (>10 cigarettes). Each patient agreed to participate in the study, providing written informed consent. The Ethics Committee of Universidad de Los Andes approved the study protocol by the Revised 
Declaration of Helsinki (64th World Medical Association General Assembly, Fortaleza, Brazil, October 2013).

\section{Surgical approach}

Preparation of dentin, L-PRF membrane and liquid fibrinogen mix (Figure 1).

Each tooth was extracted carefully, preserving the integrity of the four bony socket walls. A high-speed tungsten carbide bur was used to clean the tooth and to remove any decay, restoration or foreign materials. The tooth was dried by air and grinded with the Smart Grinder Dentin TM (Kometa Bio, Holon, Israel), according to the protocol of the manufacturer (Figure 1a-c). Dentin particles were obtained with a dimension of 300-1200 $\mu \mathrm{m}[46]$.

At the same time, L-PRF membranes were prepared. Four to six $10 \mathrm{cc}$ blood samples were collected in vacutainer tubes without anticoagulant (red cap, glass coating) and immediately centrifuged at $2700 \mathrm{rpm}$ (408g) for 12 minutes (IntraSpin, Intra-Lock, Florida, USA). Two extra blood samples were collected in $9 \mathrm{cc}$ non-coated vacutainer tubes also without anticoagulants (white cap). These last samples were centrifuged at $2700 \mathrm{rpm}$ for 3 minutes only. The yellow fluid (liquid fibrinogen) at the top of the white cap tubes was aspirated with a sterile syringe, avoiding red blood cells (Figure 1d). The L-PRF clots obtained after 12 minutes of centrifugation were placed in the Xpression box (IntraSpin, Intra-Lock, Florida, USA) for 5 minutes to gently compress (by gravity) into membranes (Figure 1e).

To prepare the L-PRF Block (Figure 1f-g), the L-PRF membranes are cut into small pieces and mixed with the dentin particles at a ratio of 2 membranes $/ 0,5 \mathrm{~g}$ dentin (which provides a 1:1 volume ratio). The liquid fibrinogen is added to the homogeneous mix, and stirred gently for \pm 10 seconds while shaping the mixture to the desired form. The fibrinogen will be converted into fibrin (primarily from activated blood platelets from the chopped L-PRF membranes) within a few minutes and trap the biomaterial and the L-PRF pieces to form a strong block, resulting in a handy and compact graft ("Dentin block") (Figure 1g).

\section{Alveolar ridge preservation}

After exhaustive cleaning, each socket was filled with the "Dentin block" shaped according to the size and shape of the socket. After adaptation of the graft to the socket, the graft was covered with two layers of L-PRF membranes extending $2 \mathrm{~mm}$ in the envelope prepared between the periosteum and the bony borders of the socket $\left(360^{\circ}\right.$ around). The wound was secured with a silk suture, not with the intention to close the wound, but simply to keep the graft and membranes stable in position. (Figure 2)

\section{Dimensional changes evaluation by CBCT.}

Horizontal and vertical measurements of alveolar ridge were evaluated by CBCT at Preextraction socket, after 4 months of alveolar ridge preservation and 6 months later of implant placement.

\section{Biopsy collection}

After a 4 to 6-months healing period, at the moment of the implant placement, a biopsy from the core of the grafted site was obtained using a $2 \mathrm{~mm}$ trephine bur. The biopsy was immediately fixed in $10 \%$ neutral buffered formalin and then dehydrated through baths of progressively more concentrated (from $50 \%$ to $100 \%$ ) alcohol, and subsequently embedded 
in paraffin. Finally, a tissue section 4- $\mu \mathrm{m}$ thick was prepared and stained with hematoxylineosin for histological analysis

\section{Histological and histo-morphometric analysis}

Two independent professionals analyzed the specimens at 10x magnification using ImageJ software. All specimens were digitized at the same magnification using a Leica DM500 microscope (Leica Microsystems, Wetzlar, Germany) and a digital camera (ICC 50 HD, Leica, Wetzlar, Germany) to measure the proportional areas of new bone, residual dentin graft particles, and connective tissue. The spatial scale of the active image was defined so measurements could be performed in calibrated units $\left(\mu \mathrm{m}^{2}\right)$. These measurements were expressed as a mean percentage of the total surface area of the section. One reviewer made a detailed histological evaluation of the image.

\section{Statistical analysis}

This paper only presents descriptive data. The histological data from both evaluators were averaged. These data are summarized in a table representing relative proportions of the different tissues (mean, median and standard deviation).

\section{Results}

A total of 10 consecutive extraction sockets ( 4 incisors, 5 canines, and 1 premolars) in the maxilla of 4 female patients (mean age 54 years; age range: 44-63 years) were included (Table 1). The reason for tooth extraction was root caries, endodontic pathology and severe periodontitis. All patients completed the study.

\section{CBCT evaluation}

4 Months later of alveolar ridge preservation, the vertical and horizontal dimensions were preserved and even increased. Furthermore, these dimensional measurements remained stable after 6 months of implant placement. (Figure 3).

\section{Clinical outcome}

The healing was always without any sign of host tissue response (inflammation or rejection symptoms), and no post-operative complications were observed. At re-entry, the grafted sites presented a homogenous appearance without disaggregated graft particles, neither in the flap, or at the entrance of the previous socket. During the implant site preparation, the hardness of the newly formed tissue was comparable to dense Type 2 bone.

\section{Histological and Histo-morphometric results}

Table 2 shows the histo-morphometric analysis of the 10 sites (one per socket) carried out by two independent evaluators. The overall mean proportion of bone was 56.5\% (S.D. 22.2\%) and of remaining dentin was 3.6\% (S.D. 6.4\%). In 4 of the 10 sites all dentin seemed to have disappeared, and in another 2 sites its proportion was $\leq 1 \%$.

When comparing the different time intervals, the relative proportion of bone increased (26.3\% at 4 months, $56.5 \%$ at 5 months, and $66.5 \%$ at 6 months, respectively). The opposite 
was seen for the amount of remaining dentin (10.4\% at 4 months, $4.8 \%$ at 5 months, and $0.9 \%$ at 6 months, respectively).

The samples in general showed the presence of compact bone tissue with normal osteocytes and with some geometric dentin fragments (5-10\% of total mineralized tissue). The bone tissue showed moderate osteoblastic activity and a medullary space filled with connective tissue showing a slight mononuclear infiltrate (Figure 4).

\section{Discussion}

In the present study, the use of a "Dentin block" in an alveolar ridge preservation model, showed be able to preserve and even slightly to increase the vertical and horizontal dimensions remaining stable after 6 months of implant placement. Additionally, was observed an optimal bone formation without host tissue response to the graft, and an appropriate rate of dentin resorption.

Different ARP techniques have been used to prevent the predictable horizontal and vertical bone resorption. Recently, Iocca and co-workers (2017) presented a meta-analysis comparing different grafting materials for ridge preservation [47]. The analysis showed that socket grafting was more clearly favorable than unassisted natural socket healing. The combination of freeze-dried bone grafts with a membrane appeared to be more efficient in the prevention of bone height resorption, whereas autologous bone marrow grafts resulted in the least resorption in ridge width [47]. However, these bone grafts have limitations related to availability and morbidity, uncertain resorption rate, cost-effectiveness, etc. [13, 14].

The "Dentin Block" is a mix of three autologous biomaterials: L-PRF, dentin and liquid fibrinogen. The ability of dentin to promote bone formation in the alveolar ridge can be explained by its common embryological origin and similar composition [21, 22]. Dentin and bone also have non-collagenous proteins belonging to the SIBLING family (Small IntegrinBinding Ligand, N-linked Glycoprotein), including dentin sialophosphoprotein (DSPP), dentin matrix protein 1 (DMP1), bone sialoprotein (BSP) and osteopontin (OPN) [48]. Moreover, BMPs, transforming growth factor-beta (TGF- $\beta$ ), insulin growth factor-I (IGF-I), and IGF-II have been detected in human dentin, and all are involved in the bone formation process [48]. Furthermore, Reis-Filho and co-workers (2012) reported that demineralized human dentin matrix (DHDM) increased the expression of vascular endothelial growth factor (VEGF), the most important factor for angiogenesis and an essential element in every healing process [8]. They also indicated that VEGF expression in the osteoblasts, stimulated by DHDM, could be necessary for the communication between osteoblast and endothelial cells during bone repair [8]. De Oliveira and co-workers (2013) evaluated histological and immunohistochemically rat alveolar wounds filled with demineralized human dentin matrix (DHDM) [49]. They observed in the experimental sites (DHDM) an advanced healing compared with the control sites (blood clot) during all the observation period; even at 14 days. The dentin was not degraded but was incorporated into trabecular bone. They also reported a statistically significant $(\mathrm{p}<0.05)$ increase in number of osteoblasts positive for BMP-2 and BMP-4 at the experimental side, compared with controls at ten days [49]. As such, dentin can be considered an absorbable matrix with osteoconductive and osteoinductive 
properties. As dentin is progressively degraded, BMPs are released in a controlled manner, stimulating bone formation [49].

Our histological analysis showed that autologous dentin was able to produce mature bone tissue. When the samples collected at different times were compared one could observe an incremental increase in the bone and at the same time a decrease in the dentin percentage. Moreover, a complete incorporation of the dentin particles into the new bone became visible with intimate contact without soft tissue in between, (resembling an ankylosis). This observation is in accordance with the results observed by de Oliveira and co-workers (2012) and Reis-Filho and co-workers (2012) [8,49]. This may confirm the biocompatibility of dentin in addition to its chemotactic characteristic for osteogenic cells due to the presence of bioactive molecules [49]. Bakhshalian and co-workers (2013) reported an increased bone mass and superior bone quality in experimental defects in rabbits filled with allogenic demineralized dentin matrix (DDM) compared with control defects (empty) [23].

An interesting clinical feature of our grafted sites was the bone quality with a hardness similar to dense trabecular (type 2). This could be related to results obtained by Kim and co-workers (2014) from scanning electron microscope (SEM) analysis where the density, roughness, and homogeneity of autogenous tooth bone graft material were relatively similar to those of autogenous cortical bone [50].

The majority of dentin studies have evaluated demineralized dentin because this process could improve the collagen exposition and delivery of BMPs and other bioactive molecules [51]. However, our results showed that non-demineralized dentin also successfully promoted bone formation with an adequate dentin resorption/bone formation rate. It is possible to speculate that the dentin cleaner kit, based on alcohols and buffer solution to eliminate all organic remains, also improved the collagen fiber exposure and the release of growth factors.

In this study the dentin had a particle size range $(300-1200 \mu \mathrm{m})$. The optimal particle size of a bone graft material depends on the type of bone substitute $[52,53]$. The morphology and size of a graft particles are important elements to consider because they determinate the vascularization, cell migration, and cell attachment, which are essential conditions to induce the new bone formation [54]. Koga and co-workers (2016) found better results with $70 \%$ dentin demineralization, and large particle size $(1000 \mu \mathrm{m})$ but proposed to analyze different degrees of dentin demineralization, for example, only surface demineralization and a mixture of particles with variable size, to obtain superior bone formation [51].

In the present study, the dentin graft was used in combination with L-PRF and liquid fibrinogen. L-PRF is the second generation of platelet concentrate obtained by a simple protocol of centrifugation (2700rpm during 12 minutes) without additives. This straightforward and inexpensive technology has shown to be able to enhance the healing process and to promote tissue regeneration due to the presence of platelets and leukocytes which release growth factors and cytokines involved in the healing process.

The particular fibrin matrix with a specific tri-dimensional architecture also favors the different biological events during tissue regeneration [40, 55-58], and enhances graft 
stability. The incorporation of small L-PRF pieces in the Dentin Block to reduce the amount of particulate graft seems to maximize the regenerative process.

The slow release of growth factors from L-PRF and their fibrin mesh gives an excellent scaffold for the migration of stem cells and osteogenic cells, possibly improving the angiogenesis and the new bone formation. The 1:1 ratio of dentin and L-PRF in the Dentin Block produces a unique tri-dimensional distribution, generating spaces between the dentin particles so that the angiogenesis and cell migration can be facilitated. The liquid fibrinogen also releases growth factors and at the same time fixes all graft components together, allowing an easy handling, avoiding the loss of graft particles, and stabilizing the regenerating tissues [45].

We chose different time points for sample collection due to the critical role of resorption rate when evaluating a bone graft. Considering that most grafting techniques suggest 4 to 6 months' period for implant placement, we adopted these intervals for sampling (4, 5 and 6 months) to get an overview of the resorption behavior of the Dentin Block graft.

The small sample size could be a limitation of our study. However, the primary objective was to obtain a preliminary view of the clinical and histological results from the use of a new graft material as a starting point for future research. Our preliminary results show promising results with an autologous graft "Dentin block" combining different regenerative properties originating from 3 biomaterials obtained from the patient himself with a low cost and a simple protocol, maximizing bone formation in a natural guided regeneration. It would be interesting in future studies to analyze the use of dentin with different degree of demineralization and to evaluate molecular aspects such as BMPs, growth factors, and cytokines released in the grafted sites in comparison to others bone substitutes. Another attractive issue would be a more detailed clinical and imaging analysis with standardized measurement methods to quantify the degree of alveolar ridge resorption. Moreover, the use of dentin block should be evaluated in more demanding regenerative scenarios (sinus lift, horizontal or vertical bone augmentation, periodontal defects, sockets with bone dehiscence, etc.).

Within the limitations of the present descriptive study, it was concluded that Dentin block was able to promote new bone formation, without host tissue reactions, and a favorable dentin resorption/bone formation rate. All patients demonstrated an adequate amount and quality of bone for implant placement. Although longer-term, multicenter, randomized, controlled clinical trials are required to confirm these observations, our finding suggests that a "Dentin block" could be a promising new substitute for bone regeneration. 


\section{Figure \& Tables Legends}

Fig. 1 Preparation \& application of Dentin Block

a. Smart Grinder Dentin TM (Kometa Bio, Holon, Israel). b. Extracted central incisor after cleaning. c. Particulate dentin as obtained at the end of process following a specific protocol (organic rest free) (particles $300 \mu \mathrm{m}-1200 \mu \mathrm{m}$ in size). d. Five L-PRF membranes after compression of the cloths in Xpression box (IntraSpin, Intra-Lock, Florida, USA). e. Liquide fibrinogen, aspirated from white cap tubes, after 3 min of centrifugation). f. Dentin block obtained after mixing $0.5 \mathrm{~g}$ dentin particles with 2 chopped L-PRF membranes and afterwards adding the liquid fibrinogen.

Fig. 2 Ridge preservation via the use of a Dentin Block

a. Extraction socket (4-wall defect) of central upper incisor (tooth 1.1). b. Dentin block graft insertion. c. Relative position of Dentin block towards marginal bone. d. Dentin block graft is covered by two layer of L-PRF membranes secured with a silk suture. e. Bone healing after 5 months, at the moment of biopsy and implant placement.

Fig. 3 CBCT comparative analyses between baseline and after 4 months

a. Vertical dimension of alveolar ridge pre-tooth extraction (tooth 1.4). b. Horizontal alveolar ridge measure pre-tooth extraction (tooth 1.4). The image $\mathrm{c}$ and $\mathrm{d}$ shows the vertical and horizontal dimensions respectively of alveolar ridge after 4 month of alveolar ridge preservation when the implant was placement. The image e and f shows the vertical and horizontal dimensions respectively of alveolar ridge after 6 month of implant placement.

Fig. 4 Histological samples (hematoxylin-eosin staining)

a. Histologic section (10x magnification) from the core of grafted site showing compact bone (black arrow), connective tissue (yellow arrow) and a remaining dentin particle (red arrow).

b. Histologic section (100x magnification) showing newly formed lamellar bone (black arrow) embedding a dentin graft particle, with clear dentinal tubules (red arrow). The yellow lines indicate the width and length of dentin particle.

Table 1. Demographic characteristic of patients.

Table 2. Relative proportions in the 10 test sockets of bone, dentin and connective tissue estimated via histo-morphometric analysis. Biopsies were taken after 4, 5 or 6 months of submucosal healing. 


\section{Compliance with Ethical Standards}

Conflict of Interest: Author Catherine Andrade declares that they have no conflicts of interest. Author Joaquin Camino declares that he has no conflict of interest. Author Mauricio Nally declares that he has no conflict of interest. Author Marc Quirynen declares that he has no conflict of interest. Author Benjamin Martinez declares that he has no conflict of interest. Author Nelson Pinto declares that he has no conflict of interest.

Funding: The present study was supported by own funds for research projects of Universidad de Los Andes.

Ethical approval: All procedures performed in the present study involving human participants were in accordance with the ethical standards of the institutional and/or national research committee and with the 1964 Helsinki declaration and its later amendments or comparable ethical standards.

Informed consent: Informed consent was obtained from all individual participants included in the study. 


\section{References}

1. Pietrokovski J, Massler M (1967) Alveolar ridge resorption following tooth extraction. J Prosthet Dent 17:21-27 . doi: 10.1016/0022-3913(67)90046-7

2. Schropp L, Wenzel A, Kostopoulos L, Karring T (2003) Bone healing and soft tissue contour changes following single-tooth extraction: a clinical and radiographic 12-month prospective study. Int J Periodontics Restorative Dent 23:313-323

3. Tan WL, Wong TLT, Wong MCM, Lang NP (2012) A systematic review of postextractional alveolar hard and soft tissue dimensional changes in humans. Clin Oral Implants Res 23:1-21 . doi: 10.1111/j.1600-0501.2011.02375.x

4. Chappuis V, Araújo MG, Buser D (2017) Clinical relevance of dimensional bone and soft tissue alterations post-extraction in esthetic sites. Periodontol 2000 73:73-83

5. Jung RE, Philipp A, Annen BM, et al (2013) Radiographic evaluation of different techniques for ridge preservation after tooth extraction: a randomized controlled clinical trial. J Clin Periodontol 40:90-98 . doi: 10.1111/jcpe.12027

6. Araújo MG, da Silva JCC, de Mendonça AF, Lindhe J (2015) Ridge alterations following grafting of fresh extraction sockets in man. A randomized clinical trial. Clin Oral Implants Res 26:407-412 . doi: 10.1111/clr.12366

7. Araújo MG, Silva CO, Misawa M, Sukekava F (2015) Alveolar socket healing: what can we learn? Periodontol 2000 68:122-134 . doi: 10.1111/prd.12082

8. Reis-Filho CR, Silva ER, Martins AB, et al (2012) Demineralised human dentine matrix stimulates the expression of VEGF and accelerates the bone repair in tooth sockets of rats. Arch Oral Biol 57:469-476 . doi: 10.1016/j.archoralbio.2011.10.011

9. Vignoletti F, Matesanz P, Rodrigo D, et al (2012) Surgical protocols for ridge preservation after tooth extraction. A systematic review. Clin Oral Implants Res 23:22-38 . doi: 10.1111/j.1600-0501.2011.02331.x

10. Willenbacher M, Al-Nawas B, Berres M, et al (2016) The Effects of Alveolar Ridge Preservation: A Meta-Analysis: Alveolar Ridge Preservation. Clin Implant Dent Relat Res 18:1248-1268 . doi: 10.1111/cid.12364

11. Giannoudis PV, Dinopoulos H, Tsiridis E (2005) Bone substitutes: An update. Injury 36:S20-S27 . doi: 10.1016/j.injury.2005.07.029

12. Morjaria KR, Wilson R, Palmer RM (2014) Bone Healing after Tooth Extraction 
with or without an Intervention: A Systematic Review of Randomized Controlled Trials: Extraction Healing with or without an Intervention. Clin Implant Dent Relat Res 16:1-20 . doi: 10.1111/j.1708-8208.2012.00450.x

13. Hallman M, Thor A (2008) Bone substitutes and growth factors as an alternative/complement to autogenous bone for grafting in implant dentistry. Periodontol 2000 47:172-192

14. Oppenheimer AJ, Mesa J, Buchman SR (2012) Current and Emerging Basic Science Concepts in Bone Biology: Implications in Craniofacial Surgery. J Craniofac Surg 23:30-36 . doi: 10.1097/SCS.0b013e318240c6d9

15. Zitzmann NU, Schärer P, Marinello CP (2001) Long-term results of implants treated with guided bone regeneration: a 5-year prospective study. Int J Oral Maxillofac Implants 16:355-366

16. Yeomans JD, Urist MR (1967) Bone induction by decalcified dentine implanted into oral, osseous and muscle tissues. Arch Oral Biol 12:999IN131007IN151006IN141008IN16

17. Moharamzadeh K, Freeman C, Blackwood K (2008) Processed bovine dentine as a bone substitute. Br J Oral Maxillofac Surg 46:110-113 . doi: 10.1016/j.bjoms.2007.07.209

18. Kim W-B, Kim S-G, Lim S-C, et al (2010) Effect of Tisseel on bone healing with particulate dentin and plaster of Paris mixture. Oral Surg Oral Med Oral Pathol Oral Radiol Endodontology 109:e34-e40 . doi: 10.1016/j.tripleo.2009.09.004

19. Kim Y-K, Kim S-G, Byeon J-H, et al (2010) Development of a novel bone grafting material using autogenous teeth. Oral Surg Oral Med Oral Pathol Oral Radiol Endodontology 109:496-503 . doi: 10.1016/j.tripleo.2009.10.017

20. Gomes MF, Valva VN, Vieira EMM, et al (2016) Homogenous demineralized dentin matrix and platelet-rich plasma for bone tissue engineering in cranioplasty of diabetic rabbits: biochemical, radiographic, and histological analysis. Int J Oral Maxillofac Surg 45:255-266 . doi: 10.1016/j.ijom.2015.09.009

21. Melek LN, El Said MM (2017) Evaluation of "Autogenous Bioengineered Injectable PRF - Tooth graft" combination (ABIT) in reconstruction of maxillary alveolar ridge defects: CBCT volumetric analysis. Saudi J Dent Res 8:86-96 . doi: 10.1016/j.sjdr.2016.10.005

22. Kim Y-K, Lee J, Um I-W, et al (2013) Tooth-derived bone graft material. J Korean Assoc Oral Maxillofac Surg 39:103 . doi: 10.5125/jkaoms.2013.39.3.103

23. Bakhshalian N, Hooshmand S, Campbell SC, et al (2013) Biocompatibility and Microstructural Analysis of Osteopromotive Property of Allogenic Demineralized 
Dentin Matrix. Int J Oral Maxillofac Implants 28:1655-1662 . doi: $10.11607 /$ jomi.2833

24. Al-Asfour A, Farzad P, Andersson L, et al (2014) Host tissue reactions of nondemineralized autogenic and xenogenic dentin blocks implanted in a nonosteogenic environment. An experimental study in rabbits. Dent Traumatol 30:198-203 . doi: 10.1111/edt.12066

25. Kim Y-K, Kim S-G, Yun P-Y, et al (2014) Autogenous teeth used for bone grafting: a comparison with traditional grafting materials. Oral Surg Oral Med Oral Pathol Oral Radiol 117:e39-e45 . doi: 10.1016/j.00oo.2012.04.018

26. Pang K-M, Um I-W, Kim Y-K, et al (2016) Autogenous demineralized dentin matrix from extracted tooth for the augmentation of alveolar bone defect: a prospective randomized clinical trial in comparison with anorganic bovine bone. Clin Oral Implants Res. doi: 10.1111/clr.12885

27. Vervelle A., Adda F., Choukroun J. An opportunity in perio-implantology: the PRF. Implantodontie

28. Dohan Ehrenfest DM, Bielecki T, Mishra A, et al (2012) In search of a consensus terminology in the field of platelet concentrates for surgical use: platelet-rich plasma (PRP), platelet-rich fibrin (PRF), fibrin gel polymerization and leukocytes. Curr Pharm Biotechnol 13:1131-1137

29. Kumar YR, Mohanty S, Verma M, et al (2016) Platelet-rich fibrin: the benefits. Br J Oral Maxillofac Surg 54:57-61 . doi: 10.1016/j.bjoms.2015.10.015

30. Singh A, Kohli M, Gupta N (2012) Platelet Rich Fibrin: A Novel Approach for Osseous Regeneration. J Maxillofac Oral Surg 11:430-434 . doi: 10.1007/s12663012-0351-0

31. Choukroun J, Adda F, Schoeffler C, Vervelle A (2001) The opportunity in perioimplantology: The PRF

32. Toffler M, Toscano N, Holtzclaw D (2010) Osteotome-Mediated Sinus Floor Elevation Using Only Platelet-Rich Fibrin: An Early Report on 110 Patients: Implant Dent 19:447-456 . doi: 10.1097/ID.0b013e3181f57288

33. Sammartino G, Ehrenfest DMD, Carile F, et al (2011) Prevention of Hemorrhagic Complications After Dental Extractions Into Open Heart Surgery Patients Under Anticoagulant Therapy: The Use of Leukocyte- and Platelet-Rich Fibrin. J Oral Implantol 37:681-690 . doi: 10.1563/AAID-JOI-D-11-00001

34. Simonpieri A, Choukroun J, Corso MD, et al (2011) Simultaneous Sinus-Lift and Implantation Using Microthreaded Implants and Leukocyte- and Platelet-Rich Fibrin as Sole Grafting Material: A Six-Year Experience: Implant Dent 20:2-12 . 
doi: 10.1097/ID.0b013e3181faa8af

35. Simonpieri A, Del Corso M, Vervelle A, et al (2012) Current knowledge and perspectives for the use of platelet-rich plasma (PRP) and platelet-rich fibrin (PRF) in oral and maxillofacial surgery part 2: Bone graft, implant and reconstructive surgery. Curr Pharm Biotechnol 13:1231-1256

36. Bielecki T, Dohan Ehrenfest DM (2012) Leukocyte- and platelet-rich Plasma (LPRP)/fibrin (L-PRF) in medicine - past, present, future. Curr Pharm Biotechnol 13:i-ii

37. Zumstein MA, Rumian A, Lesbats V, et al (2014) Increased vascularization during early healing after biologic augmentation in repair of chronic rotator cuff tears using autologous leukocyte- and platelet-rich fibrin (L-PRF): a prospective randomized controlled pilot trial. J Shoulder Elbow Surg 23:3-12 . doi: 10.1016/j.jse.2013.08.017

38. Tunali M, Ozdemir H, Arabaci T, et al (2015) Clinical Evaluation of Autologous Platelet-Rich Fibrin in the Treatment of Multiple Adjacent Gingival Recession Defects: A 12-Month Study. Int J Periodontics Restorative Dent 35:105-114 . doi: $10.11607 /$ prd.1826

39. Castro AB, Meschi N, Temmerman A, et al (2017) Regenerative potential of leucocyte- and platelet-rich fibrin. Part A: intra-bony defects, furcation defects and periodontal plastic surgery. A systematic review and meta-analysis. J Clin Periodontol 44:67-82 . doi: 10.1111/jcpe.12643

40. Castro AB, Meschi N, Temmerman A, et al (2017) Regenerative potential of leucocyte- and platelet-rich fibrin. Part B: sinus floor elevation, alveolar ridge preservation and implant therapy. A systematic review. J Clin Periodontol 44:225-234 . doi: 10.1111/jcpe.12658

41. Chignon-Sicard B, Georgiou CA, Fontas E, et al (2012) Efficacy of Leukocyte- and Platelet-Rich Fibrin in Wound Healing: A Randomized Controlled Clinical Trial. Plast Reconstr Surg 130:819e-829e . doi: 10.1097/PRS.0b013e31826d1711

42. Jankovic S, Aleksic Z, Klokkevold P, et al (2012) Use of platelet-rich fibrin membrane following treatment of gingival recession: a randomized clinical trial. Int J Periodontics Restorative Dent 32:e41-50

43. Kumar N, Prasad K, Ramanujam L, et al (2015) Evaluation of Treatment Outcome After Impacted Mandibular Third Molar Surgery With the Use of Autologous Platelet-Rich Fibrin: A Randomized Controlled Clinical Study. J Oral Maxillofac Surg 73:1042-1049 . doi: 10.1016/j.joms.2014.11.013

44. Temmerman A, Vandessel J, Castro A, et al (2016) The use of leucocyte and platelet-rich fibrin in socket management and ridge preservation: a split-mouth, 
randomized, controlled clinical trial. J Clin Periodontol 43:990-999 . doi: $10.1111 /$ jcpe. 12612

45. Andrade C, Orrego A, Pinto N (2016) HEMATOLOGIC PROFILE OF FOUR AUTOLOGOUS FIBRINOGEN BASED BINDER OBTAINED WITH DIFFERENTS PROTOCOLS

46. Binderman I, Hallel G, Nardy C, et al (2015) Processing extracted teeth for immediate grafting of autogenous dentin. Implant Pract US 2:43-46

47. Iocca O, Farcomeni A, Pardiñas Lopez S, Talib HS (2017) Alveolar ridge preservation after tooth extraction: a Bayesian Network meta-analysis of grafting materials efficacy on prevention of bone height and width reduction. J Clin Periodontol 44:104-114 . doi: 10.1111/jcpe.12633

48. Murata M, Akazawa T, Mitsugi M, et al (2013) Autograft of Dentin Materials for Bone Regeneration. In: Pignatello R (ed) Advances in Biomaterials Science and Biomedical Applications. InTech

49. de Oliveira G, Miziara M, Silva E da, et al (2013) Enhanced bone formation during healing process of tooth sockets filled with demineralized human dentine matrix. Aust Dent J 58:326-332 . doi: 10.1111/adj.12088

50. Kim Y-K, Yun P-Y, Um I-W, et al (2014) Alveolar ridge preservation of an extraction socket using autogenous tooth bone graft material for implant site development: prospective case series. J Adv Prosthodont 6:521 . doi: 10.4047/jap.2014.6.6.521

51. Koga T, Minamizato T, Kawai Y, et al (2016) Bone Regeneration Using Dentin Matrix Depends on the Degree of Demineralization and Particle Size. PLOS ONE 11:e0147235 . doi: 10.1371/journal.pone.0147235

52. Al Ruhaimi KA (2001) Bone graft substitutes: a comparative qualitative histologic review of current osteoconductive grafting materials. Int J Oral Maxillofac Implants 16:

53. Ryabenkova Y, Pinnock A, Quadros PA, et al (2017) The relationship between particle morphology and rheological properties in injectable nanohydroxyapatite bone graft substitutes. Mater Sci Eng C 75:1083-1090 . doi: 10.1016/j.msec.2017.02.170

54. Hutmacher DW, Schantz JT, Lam CXF, et al (2007) State of the art and future directions of scaffold-based bone engineering from a biomaterials perspective. J Tissue Eng Regen Med 1:245-260 . doi: 10.1002/term.24

55. Dohan DM, Choukroun J, Diss A, et al (2006) Platelet-rich fibrin (PRF): A secondgeneration platelet concentrate. Part I: Technological concepts and evolution. 
Oral Surg Oral Med Oral Pathol Oral Radiol Endodontology 101:e37-e44 . doi: 10.1016/j.tripleo.2005.07.008

56. Dohan DM, Choukroun J, Diss A, et al (2006) Platelet-rich fibrin (PRF): A secondgeneration platelet concentrate. Part II: Platelet-related biologic features. Oral Surg Oral Med Oral Pathol Oral Radiol Endodontology 101:e45-e50 . doi: 10.1016/j.tripleo.2005.07.009

57. Dohan DM, Choukroun J, Diss A, et al (2006) Platelet-rich fibrin (PRF): A secondgeneration platelet concentrate. Part III: Leucocyte activation: A new feature for platelet concentrates? Oral Surg Oral Med Oral Pathol Oral Radiol Endodontology 101:e51-e55 . doi: 10.1016/j.tripleo.2005.07.010

58. Dohan Ehrenfest DM, Del Corso M, Diss A, et al (2010) Three-Dimensional Architecture and Cell Composition of a Choukroun's Platelet-Rich Fibrin Clot and Membrane. J Periodontol 81:546-555. doi: 10.1902/jop.2009.090531 

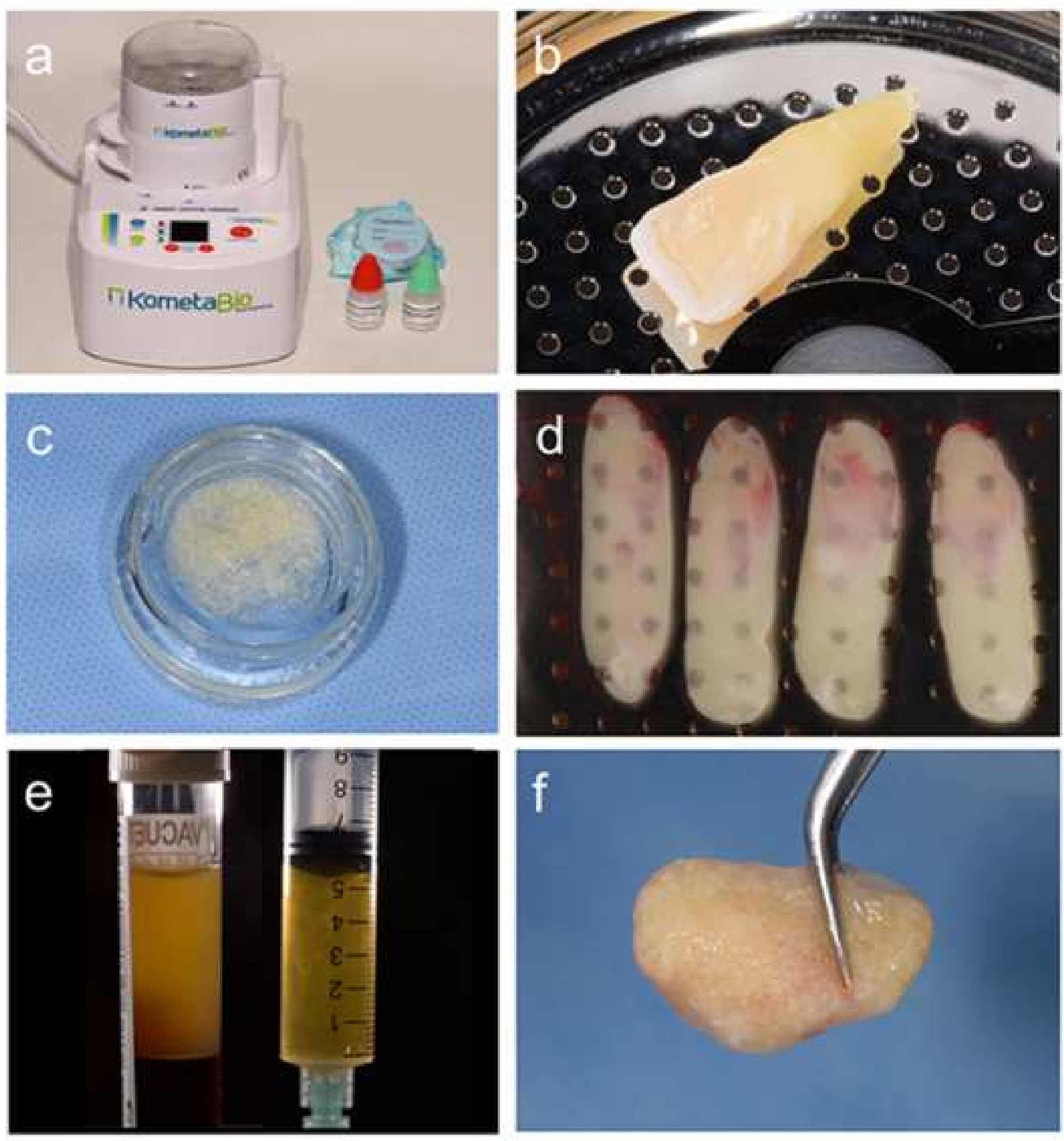


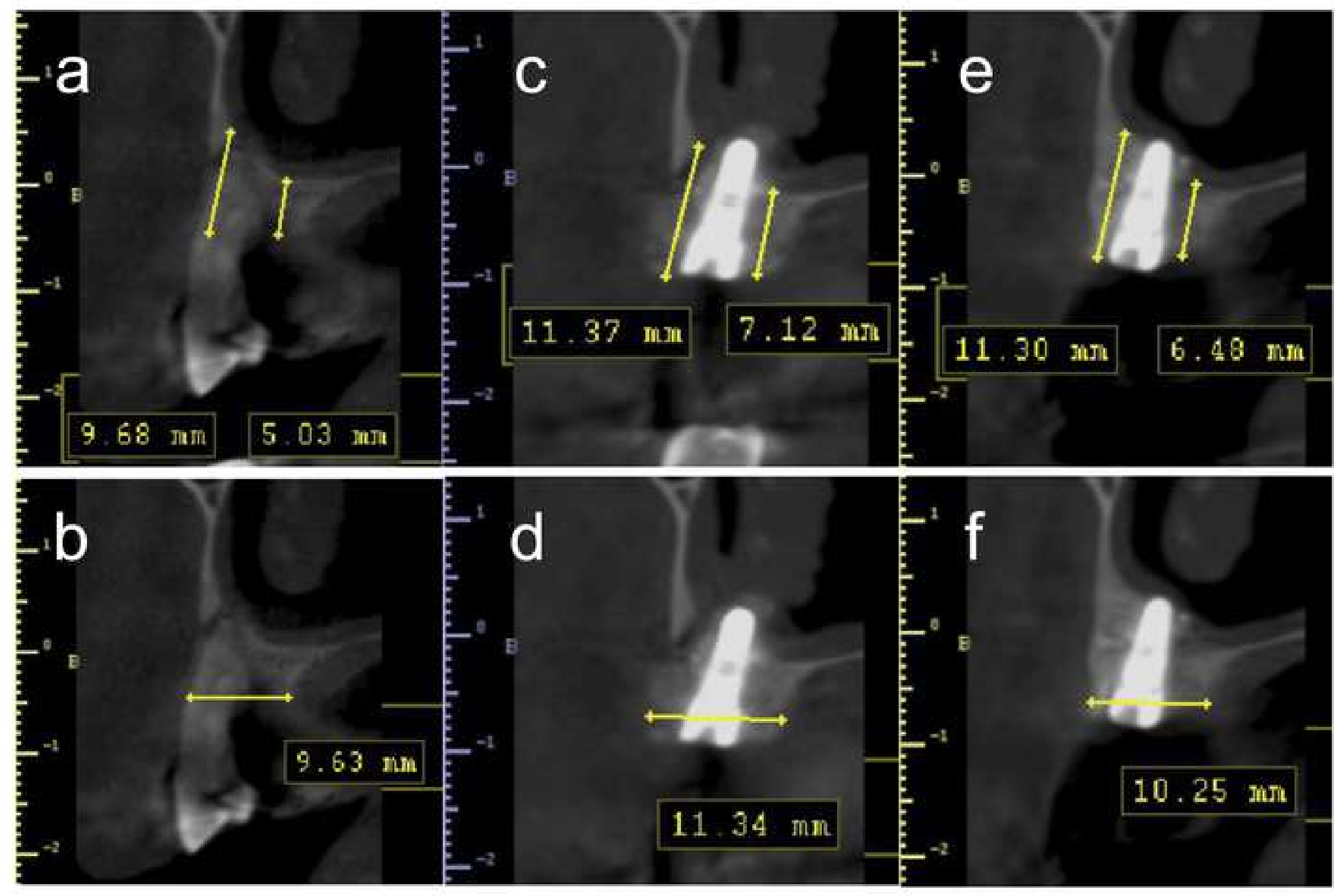



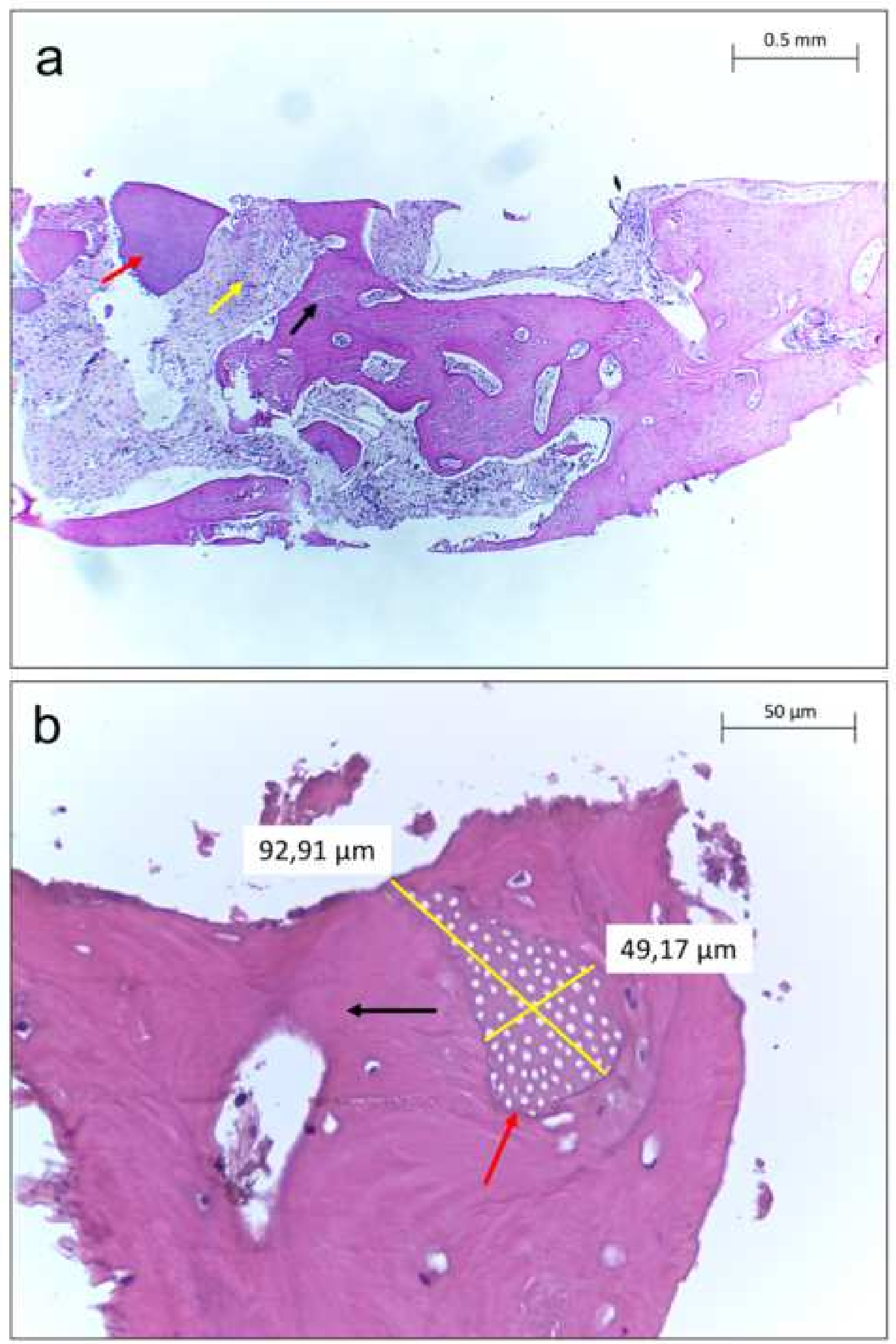
Table 1. Demographic characteristic of patients.

\begin{tabular}{ccc}
\hline Variable & Tooth site $(\mathbf{N}=\mathbf{1 0})$ \\
& & \\
& & \\
\hline Gender & Male & 0 \\
& Female & 4 \\
\hline Age & Mean & 54 \\
& Range & $44-63$ \\
\hline Tooth site location & Incisor & 4 \\
& Canine & 5 \\
& Premolar & 1 \\
\hline
\end{tabular}


Table 2. Relative proportions in the 10 test sockets of bone, dentin and connective tissue estimated via histo-morphometric analysis. Biopsies were taken after 4, 5 or 6 months of submucosal healing.

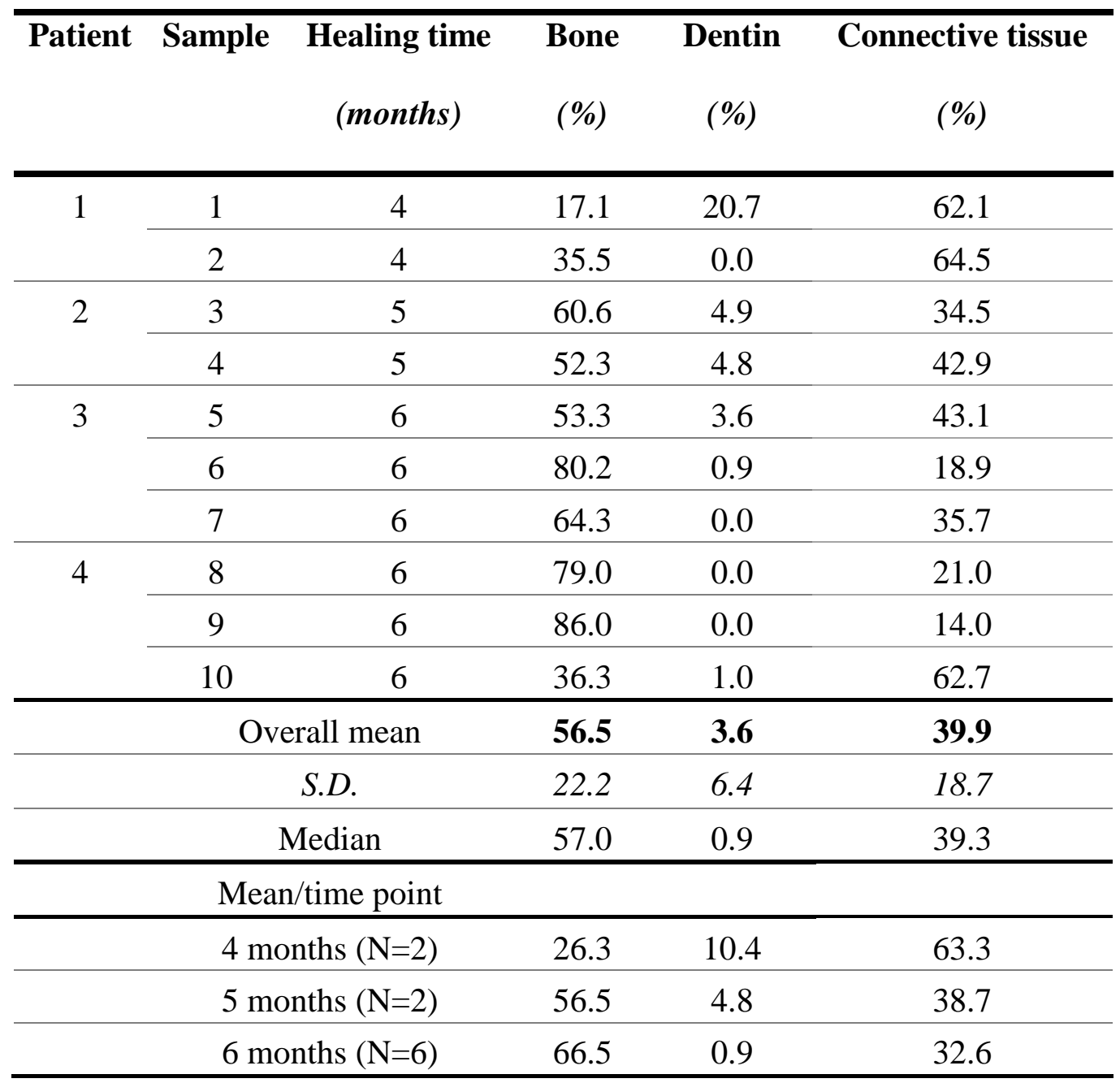

$S . D=$ Standard deviation 
Reply to reviewer: \#2

1. Concern of the reviewer: The title should clearly state that this a pilot study. Our response: The title was changed according to the requested.

Revised text: Combining autologous particulate dentin, L-PRF and fibrinogen to create a matrix for predictable ridge preservation: A pilot clinical study.

2. Concern of the reviewer: Given that is well known that the ridge preservation do not improve the bone quality nor the implant success, given that the only advantage seems to be related to the maintenance of the tissue volume after a tooth extraction, the authors should include a volumetric and radiologic evaluation to obtain clear data on the real clinical impact of this technique. The authors should comment that address an answer and include some clinical data.

Our response: To clarify this item was incorporated in material and methods, results and discussion new information related to the dimensional changes of alveolar ridge.

Revised text:

In Material and methods:

Dimensional changes evaluation by СBCT.

Abstract section, Objectives: the authors should change the sentence, what does it mean an alveolar ridge model??' change and clarify the sentence.

extraction socket, after 4 months of alveolar ridge preservation and 6 months later of implant placement.

In Results:

CBCT evaluation

4 Months later of alveolar ridge preservation, the vertical and horizontal dimensions were preserved and even increased. Furthermore, these dimensional measurements remained stable after 6 months of implant placement. (Figure 3).

In Discussion:

In the present study, the use of a "Dentin block" in an alveolar ridge preservation model, showed be able to preserve and even slightly to increase the vertical and horizontal dimensions remaining stable after 6 months of implant placement. Additionally, was observed an optimal bone formation without host tissue response to the graft, and an appropriate rate of dentin resorption.

Furthermore, the figure 3 was changed.

3. concern of the reviewer: Abstract section, Objectives: the authors should change the sentence, what does it mean an alveolar ridge model??' change and clarify the sentence. Our response: To avoid confusion, the "model" word was deleted.

Revised text: The aim of this study was to describe the histological and clinical outcome of "Dentin block" (a mixture of: autologous particulate dentin, leukocyte and platelet rich fibrin (L-PRF) and liquid fibrinogen) in an alveolar ridge preservation.

4. concern of the reviewer: Abstract section, Mat \& met: it is not clear how many samples were taken at 4, 5 and 6 months. 
Our response: This item was clarified changing the sentence.

Revised text: 2 grafted sites were followed at 4 and 5 months, and 6 sites at 6 months.

5. concern of the reviewer: Abstract section, Results: the authors should report at least the most important outcomes

Our response: To complete the information was added a new sentence.

Revised text: The vertical and horizontal dimensions of alveolar ridge were preserved or even increased after 4, 5 or 6 months and remained stable after 6 months of the implant placement.

6. concern of the reviewer: Introduction section, pag 3, line 18: what materials are the authors talking about ?? the only biomaterial that possess these proprerties is the autogenous bone!! Please check the sentence and make changes.

Our response: In this sentence we are referring the different bone grafts available, including the autogenous bone. Mentioned that these biomaterials could have one or more of this biological properties.

To clarify this point, the sentence was changed in the text.

Revised text: These materials are used because they can possess one or more of these biological properties: osteogenesis, osteoinduction and/or osteoconduction.

7. concern of the reviewer: Introduction section, pag 3, line 50: the real advantage of ARP procedures are not related to histological outcomes but to the dimensional changes. As consequence, the authors should comment and re-write the sentence.

Our response: The sentence was re-write in the text.

Revised text: In ARP, dentin has shown be able to preserved the dimensions of alveolar ridge due to osteo-conductive property and its favorable rate of resorption and new bone formation

8. concern of the reviewer: Introduction section, pag 4, line 11-12: the sentence is not clear and it should be re-written.

Our response: The sentence was changed to clarify the concept.

Revised text: Several medical disciplines have used L-PRF in different types of surgery, including periodontology, implant dentistry and maxillofacial surgery, reporting a positive effect for the wound healing process [32-41].

9. concern of the reviewer: Mat and met section, page 4, line 52-53: what about the other anti-resorptive medications? why did the authors exclude only bisphosphonate? why pregnancy should be considered an exclusion criteria? As consequence did the authors plan any test to evaluate pregnancy during the patient's enrollement phase? In the case the periodontally affected teeth are not excluded, how did the authors plan to treat the risk of having bacteria at the level of dentin tubules? Each of the points addressed need to be discussed and relative changes reported in the manuscript text. 
Our response: Considering and appreciating your comments, we did some changes in the text in relation to the bisphosphonate. The pregnancy was a exclusion criteria by the risk of these patients of interrupt or not complete the study follow-up period. In this study this condition was just auto-reported. Related to the disinfection of the dentin tubules, the protocol incorporates the use of a disinfection kit (solutions) which has demonstrated be enough to eliminate the organic components of dentin and including bacteria.

Revised text: antiresorptive therapy (as bisphosphonates)

10. concern of the reviewer: Mat and Met section: the use of a demographic table could probably improve the comprehension of the readers. Did the re-entry time plan a distribution according to the tooth site? If the authors re-entered esthetic sites at 6 months and premolar or molar at 4 this could have an impact at the clinical level. This is not clarified and should be addressed by the authors Mat and met section, page 4: Ethical Committee, could the authors provide the acceptance of the Ethical Committee? Is the use of dentin block procedure accepted? Our response: We added it a demographic table as you suggested (Tabla 1), for that we had to change the order of tables. The changes on text are in blue. And the new table was added.

The re-entered time plan was not associated to the tooth site. The different times were considered to evaluate the dentin resorption/bone formation rate, considering the common range of time to implant placement after a bone graft (4-6 months)

The present study has the approval of ethical committee; this was accepted due the different component of dentin block have scientific evidence to be used safety and they have shown benefits to the bone formation process.

Revised text: A total of 10 consecutive extraction sockets ( 4 incisors, 5 canines, and 1 premolars) in the maxilla of 4 female patients (mean age 54 years; age range: $44-63$ years) were included (Table 1).

Table 2 shows the histo-morphometric analysis of the 10 sites (one per socket) carried out by two independent evaluators.

11. concern of the reviewer: Results section: it should contains informations on how many and what sites were re-entered at 4, 5 and 6 months. Furthermore, clinical outcomes on volumetric or radiological parameters should be added in the manuscript

Our response: On the table 2 was describe how many sites were re-entered at 4, 5 and 6 months. On results a volumetric analysis by CBCT was added.

Revised text: See table 2.

\section{CBCT evaluation}

4 Months later of alveolar ridge preservation, the vertical and horizontal dimensions were preserved and even increased. Furthermore, these dimensional measurements remained stable after 6 months of implant placement. (Figure 3). 
1. concern of the reviewer: This paper evaluates the "dentin block" for ridge preservation. It is a small proof of principle study that appears to be well done. I would suggest that the use of English is carefully proofread. Also for all the materials and equipment please make sure that the manufacturer is clearly detailed. For table I I would suggest including the tooth and patient. One issue with the use of dentine is bacterial contamination. Are the authors sure that their cleansing protocol removed all bacteria? No ridge preservation technique will stop resorption of the buccal plate. What is important is the maintenance of as much width and height as possible with good bone formation. whilst not providing a better alternative than existing methods the dentine block certainly appears no less successful. Larger and more long term studies would be very interesting.

Our response: According to your suggestions the English was reviewed and was checked that the manufacturer information for all equipment and materials used was incorporated in the text.

The table 2 was changed, adding the number of patient for the tooth sites analyzed. Relative to bacterial contamination of dentin the kit used for this propose is enough to eliminate this kind of contamination. This was probed before analyzing in a specialized laboratory of quality control.

On the other side, the use of dentin block in ridge preservation was use to show how this biomaterial is able to promote an adequate new bone formation. Suggestion that is an option for different kinds of bone regeneration techniques.

Revised text: See the changes in the table 2. 